\title{
DESIGN AND FABRICATION OF AN 805 MHZ RF CAVITY WITH BE WINDOWS FOR A HIGH RF POWER TESTING FOR A MUON COOLING EXPERIMENT*
}

\author{
Derun Li ${ }^{\square}$, J. Corlett, R. MacGill, R. Rimmer, LBNL, 1 Cyclotron Road, Berkeley, CA 94720, USA \\ D. Summers, M. Booke, M. Reep, University of Mississippi, University, MS 38677, USA
}

\section{Abstract}

We report the design and fabrication of an $805 \mathrm{MHz} \mathrm{RF}$ cavity with beryllium (Be) windows for a muon cooling experiment. The cavity resembles closely to a cylindrical pillbox cavity with the conventional beam iris covered by thin Be foils to enhance on-axis accelerating fields. The Be windows are bolted to the cavity body to allow for easy replacement and testing for different windows. The cavity is to be installed within a super-conducting solenoid with magnetic field up to 5 Tesla, and tested at high RF power.

\section{INTRODUCTION}

High gradient RF cavities are required in muon cooling channels for the design of a neutrino factory or a muon collider. A generic ionization cooling channel consists of absorbers with low $\mathrm{Z}$ material (liquid hydrogen is proposed), a high gradient linac structure (or a few RF cavities) to replenish muon energy lost in the absorbers, and alternating super-conducting solenoid to confine the muons in the channel. Due to the presence of strong magnetic fields from the superconducting solenoids, option for using high gradient superconducting RF cavity is excluded. Depending on at which cooling stages, high gradient RF cavities are needed in frequencies ranging from $201 \mathrm{MHz}$ to $805 \mathrm{MHz}$, at gradients of $15 \mathrm{MV} / \mathrm{m}$ and $30 \mathrm{MV} / \mathrm{m}$, respectively. We propose using conventional copper cavity with open beam iris covered by thin conducting foils to increase the cavity shunt impedance taking the penetration property of muon beams. Beryllium due to its low $\mathrm{Z}$ and good electric and thermal properties, makes it a perfect candidate for the thin conducting foil. Tremendous efforts have been put to study the thermal, electric, and mechanic properties of thin Be foils for application in a RF cavity [1]. R\&D has been concentrated on the design and analysis of robust RF structure with the Be foils which will operate under high electromagnetic RF fields. The Be foils are exposed to these high EM fields, and RF currents are induced in the foils which lead to temperature gradients within the foils. The foils may be deformed due to the temperature gradients, and result in detuning of the cavity so that RF power is being reflected if the RF heating can not be conducted away. Experimental programs have been devoted to study this effect, a low power $805 \mathrm{MHz}$ test cavity with Be windows was built, and a high power test facility, Lab G at Fermilab is developed for part of this purpose. This paper presents the design and fabrication of an $805 \mathrm{MHz}$ high power test cavity with Be foils. This cavity is being fabricated at the University of Mississippi, and is ready for tuning and will be tested at the high power test facility, the Lab G in Fermilab this summer.

\section{THE CAVITY DESIGN}

The high power test cavity resembles closely to a cylindrical pillbox cavity as shown in Figure 1. Two conventional irises are covered by thin Be windows. RF power is fed in through a kidney shaped coupling slot on the cavity wall where a kidney shaped transition waveguide (coupler) is attached. The coupler is jointed to a quarter wavelength rectangular waveguide before being transitioned to a standard WR975 waveguide. Joints between cavity body, coupler, and waveguides are held by non-magnetic stainless steel bolts. Tin seals are used between these joints to ensure good RF contacts and high vacuum. Design of the cavity and coupler are simulated using the MAFIA in frequency and time domains, respectively. The coupler is designed to be at critical coupling at $805 \mathrm{MHz}$. Limited by the warm bore size (44 $\mathrm{cm}$ in diameter) of the superconducting solenoid, the kidney shaped coupler is employed for leaving more space to accommodate the Be window near the coupler. Coupling calculation is carried out using the MAFIA in the time domain. Some relevant parameters of the cavity are listed in Table 1.

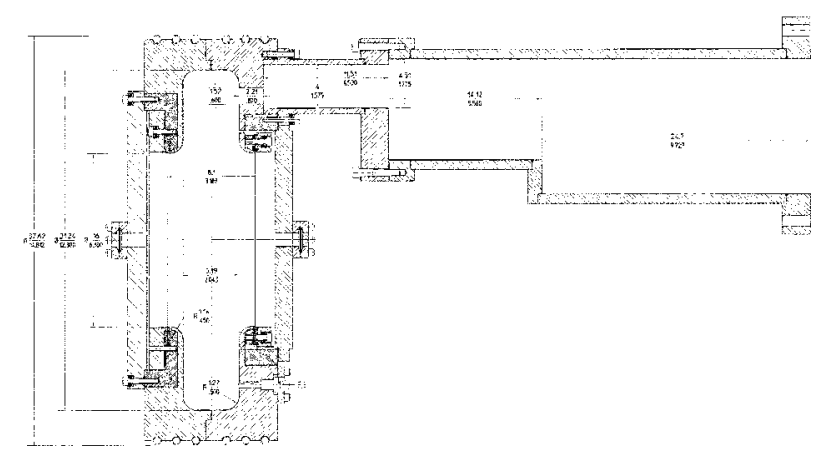

Figure 1: A cross-section view of the high power pillbox test cavity with Be windows, the coupler and transition

\footnotetext{
* Work supported by the U.S. Department of Energy under contracts No.s DE-AC0376SF00098 (LBNL), DE-FG0291ER40622 (Univ. of Mississippi)

口Email: Dli@lbl.gov
} 
waveguide, water cooling channels around the peripheral of the cavity are displayed as well.

Table 1: Parameters of the high power test cavity

\begin{tabular}{|l|c|}
\hline Frequency & $805 \mathrm{MHz}$ \\
\hline Be window thickness & $127 \mu \mathrm{m}$ \\
\hline Shunt impedance $\left(\mathrm{V}^{2} / \mathrm{P}\right)$ & $38 \mathrm{M} \Omega / \mathrm{m}$ \\
\hline $\mathrm{Q}_{0}$ & 21,000 \\
\hline Cavity diameter & $31.24 \mathrm{~cm}$ \\
\hline Cavity cell length & $8.1 \mathrm{~cm}$ \\
\hline Be window diameter & $16 \mathrm{~cm}$ \\
\hline Coupling Coefficient & 1 \\
\hline
\end{tabular}

\section{THE CAVITY FABRICATION}

The cavity body is divided into two halves, and made from OFHC. The two halves will be brazed together in hydrogen oven after final tuning. The windows are made from high purity Be foils (IF-1, $99 \%$ purity Be). Each foil is sandwiched in between two annular Be frames with less purity (PS-200, 98.5\%) by diffusion bonding (braze). Pre-tension is needed in keeping the foils flat, and is naturally induced from the brazing process. However, the exact amount of pre-tension is unknown, and difficult to measure and simulate. Measurements and modeling of the Be windows with pre-tension have been conducted in [24]. Figure 2 shows a $16 \mathrm{~cm}$ diameter, $127 \mu \mathrm{m}$ thick Be window made by Brush-wellman, a company in Fremont, California.

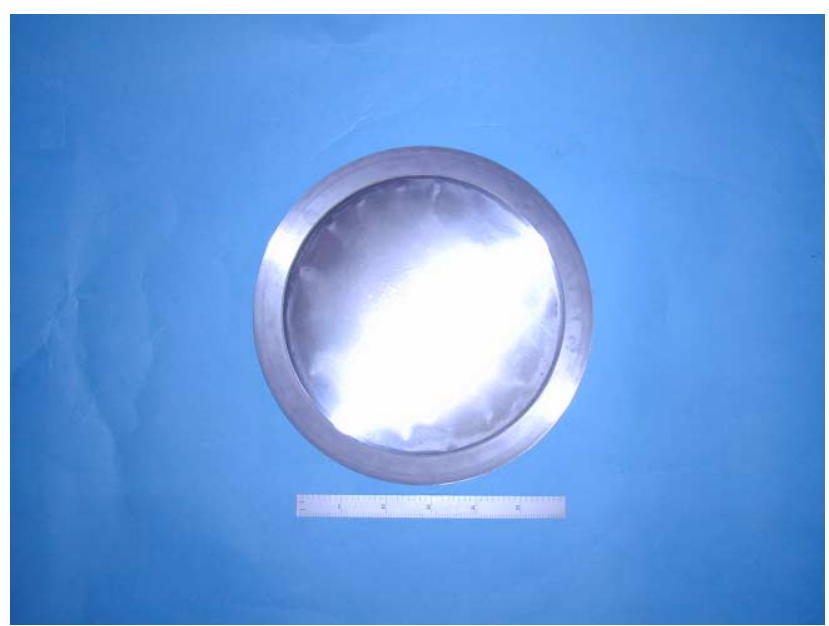

Figure 2: The Be window: a $127 \mu \mathrm{m}$ thickness Be foil (IF-

1 ) is sandwiched between two annular Be frames (PS-

200), the foil diameter is $16 \mathrm{~cm}$. This window has been used in the test of an $805 \mathrm{MHz}$ low power cold-test cavity.

To ensure good RF and thermal contacts between the Be windows and the cavity body for the high power test, three existing Be windows have to be modified. 24 holes have been added (EDM) on the annular frames to allow for a tight-hold of the windows by bolts, and as well as for easy replacement of the windows. The cavity body also needs to be stiff enough to allow for multiple changes of the windows and enduring strong torque forces from the bolts. Two stainless steel rings are brazed into the two halves of the copper body separately, and allow for more and uniform torque forces to be exerted through the bolts. One of the stainless steel rings is shown in Figure 3.

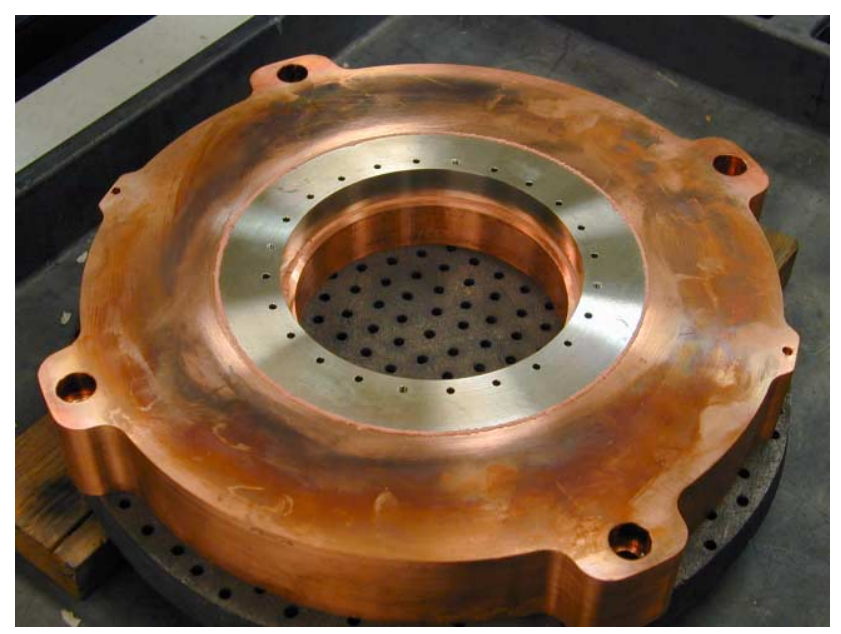

Figure 3: A stainless ring is brazed into the copper body of each half of the cavity. This photo shows the s.s. ring before it was put into hydrogen oven at Alpha-Braze a company in Fremont, California.

The two halves of the cavity are machined to their final design shapes after the stainless steel rings are brazed. Figure 4 is a photo showing the cavity is being fabricated at the University of Mississippi. The kidney shaped coupler can also be seen in the photo. Three view ports that are equally spaced and added on the outside-wall of the cavity, which will be used for visually monitoring and inspecting the windows using a bore-scope during high RF power conditioning and tests. Three antenna probes are mounted on one side of the end-plate to measure the RF power and the frequency in the cavity.

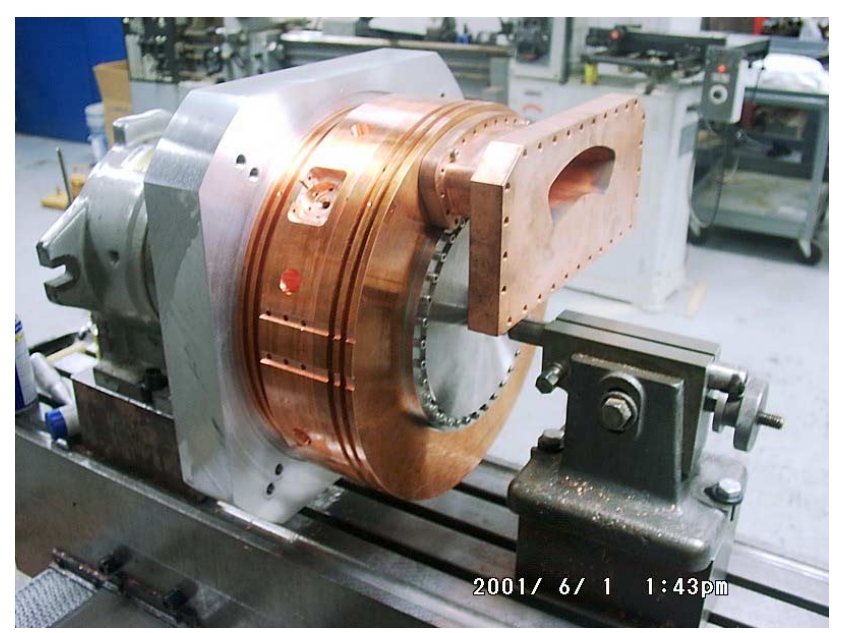

Figure 4: The single cell pillbox cavity is being fabricated at the Univ. of Mississippi. This photo shows the copper cavity body attached with the kidney shaped coupler. 
Two small compartments (small cavities) are needed behind the Be windows facing the end-plates. Vacuum channels are drilled to equalize the pressure between the cavity and the compartments so that there is no differential pressure on the Be windows. As shown in Figure 1, there are two additional ports located in the center of the end-plates. These ports can be used as regular view ports for inspecting the windows, and RF probes to monitor frequency changes of the compartment due to the window deformation by the RF heating in the cavity.

\section{THE CAVITY AND COUPLER TUNING}

It is not practical to measure the frequency at the operating temperature and under vacuum. The cavity and coupler will be tuned at room temperature and atmospheric pressure at the University of Mississippi. The cavity and the coupler then may need to be tuned to different frequency and coupling to account for the effects of temperature, strong magnetic fields, and possible extra $\mathrm{RF}$ power losses between joints of the Be windows and the cavity body. Since the high power test is for a single cell cavity, the klystron frequency can always be adjusted to match the cavity frequency within its bandwidth, which eases the cavity tuning to some extent. However, possible cavity distortion due to the final braze will not be known when the cavity is being tuned. We expect the weld shrinkage is well within the range of the klystron bandwidth. The cavity has been made with plenty of excess materials on the cavity length. The cavity tuning will be conducted by shortening the cavity length (one of the half cells), that should shift the cavity to higher frequencies. Without tuning, the cavity frequency should be measured to be near $800 \mathrm{MHz}$. The MAFIA simulation shows that the tuning sensitivity of the cavity is approximately $1.2 \mathrm{MHz} / \mathrm{mm}$. The cavity should be assembled and measured carefully before the first tuning cut. The increment of the cuts should be small to avoid over shooting. We expect to have at least 4 cuts before the final frequency $805 \mathrm{MHz}$ is reached. This is necessary for finding reference and calibrating tuning sensitivity. Coupler will be tuned at the same time while tuning the cavity. We do not expect the coupling be too sensitive within the frequency range. The coupler has been made longer than it should be based on the MAFIA simulation in the time domain. The coupling can be increased by shortening the depth of the kidney shaped waveguide. It is hard to predict the $\mathrm{Q}_{0}$ before the final braze, considering many joints in the cavity, different windows and the strong magnetic fields at operation, the coupler will be tuned using two copper windows to be slightly over coupled.

\section{HIGH POWER TEST PLAN}

The layout for the high power test at the Lab $G$ in Fermilab is shown in Figure 5. A $10 \mathrm{MW}$ peak power klystron at frequency of $805 \pm 2 \mathrm{MHz}$ is available for the test.

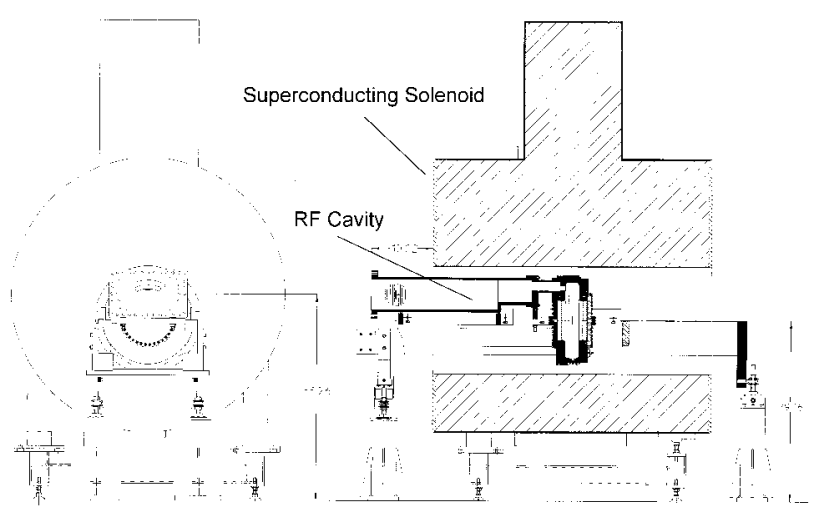

Figure 5: Layout of the high power test of the $805 \mathrm{MHz}$ pill box cavity at the Lab G in Fermilab.

The Lab $\mathrm{G}$ is dedicated for the high power test of 805 MHz RF cavity. RF power transmission line (waveguide), cooling water, vacuum pumps have been experimented and ready for the test. The superconducting solenoid has been powered and tested. A six-cell $805 \mathrm{MHz}$ open iris cavity made by Fermilab is currently being tested [1].

As shown in Figure 5, the pillbox cavity will be seating on a support rack inside the bore of the superconducting solenoid. The superconducting solenoid has two coils and can be powered independently operating at either solenoid mode and gradient mode. This will allow us for studying multipacting effects under different magnetic fields. The RF power, repetition rate, pulse length from the klystron can be adjusted to allow us for conditioning the cavity, and for searching the highest tolerable and achievable peak RF field and average heating power on the Be foils. As a comparison test, two copper windows, two additional Be windows with different thickness have been fabricated and purchased to study the cavity detuning caused by RF heating on these windows. All the Be windows are coated with $\mathrm{TiN}$ on one side to restrain multipacting, and these windows can also be flipped over for comparison tests. To investigate the multipacting effects, these Be windows will be tested at different $R F$ powers under different magnetic fields. The window deformation due to the RF heating will be studied for different window thickness with and without TiN coatings.

\section{REFERENCES}

[1] "High-Gradient normal conducting RF structures for muon cooling channels", J. Corlett, et al, This conference

[2] "Be window studies at room temperature", $\mathrm{D}$. Li, et al, MUCOOL Note 110.

[3] "Thin Beryllium Windows - Analysis and Design Status", N. Hartman, et al, NUCOOL Note 180

[4] "Temperature Distribution Calculations on Beryllium Windows in RF Cavities for a Muon Collider", D. Li, et al, LINAC-1998, Chicago, IL, U.S.A. 